\title{
Intraventricular T3 Reverses Chronic Restraint Stress-Induced Depressive-Like Behaviors: Inhibition of NF-KB/ NLRP3 Inflammasome Pathway in the Hippocampus
}

\section{Tahmineh Mokhtari}

Chinese Academy of Sciences

AymanEl-Meghawry El-Kenawy ( $\sim$ elkenawy@tu.edu.sa )

Taif University https://orcid.org/0000-0002-0818-2393

Li Hu

Chinese Academy of Sciences

Original Article

Keywords: Triiodothyronine, NF-kB, Inflammasome, NLRP3, Depression, Stress

Posted Date: February 5th, 2021

DOI: https://doi.org/10.21203/rs.3.rs-187105/v1

License: (c) (1) This work is licensed under a Creative Commons Attribution 4.0 International License.

Read Full License 


\section{Abstract}

In this study, the effects of triiodothyronine (T3) were evaluated on the NLRP3 inflammasome complex formation in the hippocampus of the rat with restraint stress-induced depressive-like behaviors. Thirty-six Wistar male rats were randomly allocated to the following groups: Control, Model, and Model + T3. In Model or Model + T3 group, a single dose of PBS or T3 0 was administered into the lateral ventricle. Depressive-like behaviors were induced by chronic restraint stress. The forced swimming (FST), tail suspension (TST), and open field (OFT) tests were used to investigate depression. The rats were sacrificed, and brain tissues were stored for molecular and pathological evaluations. Chronic stress increased the immobility of rats in the Model group according to FST, TST, and OFT $(P<0.05)$. T3 significantly improved depressive-like behaviors $(P<0.05)$. The gene expression and protein level of hippocampal NF-KB, NLRP3, ASC, and Caspase-1 significantly increased in the Model group compared to the control group $(P<0.05)$. The reduced hippocampal levels of NF-KB, NLRP3, ASC, and Caspase-1 were seen in the T3 group compared to the Model group $(P<0.05)$. Also, the Nissl staining of the CA1 region showed an increased number of dark neurons $(P<0.05)$ and reduced pyramidal layer thickness $(P<0.05)$ in the Model group. These histopathological alterations were changed by T3 administration compared to the Model group $(\mathrm{P}<0.05)$. The findings of confirmed the therapeutic effects of intraventricularly T3 on depressive-like behaviors induced by restraint stress via surviving pyramidal neurons of the CA1 region and inhibition of NF-KB/NLRP3 inflammasome pathway.

\section{Introduction}

The function of several organs and systems, especially the central nervous system (CNS) and the endocrine system, can be affected by unpredictable and undesirable stressful conditions. Chronic and long-term stress contributes to the induction of a wide range of mental disorders, e.g., anxiety and depression [1]. Depression is an alteration in cognitive behavior and mood, featured by cognitive dysfunction, pleasure loss, negative mood, social isolation fatigue, sleep disorders, appetite loss, and other metabolic changes [2,3]. Among different regions of brain, it has been shown that reduced volume of hippocampus is strongly correlated with the severity of depression [4]

Long-term unpredicted and repeated stress triggers a cascade of inflammatory responses in the brain [5]. There is a growing body of evidence from preclinical and clinical studies indicate that depression is attributed to the higher levels of cytokines, such as interleukin (IL)-6, IL-1 $\beta$ in blood and CSF [6]. The elevated levels of inflammatory cytokines, such as tumor necrosis factor-alpha (TNF-a), IL-1 $\beta$, and IL-6 in brain regions, including the hypothalamus, hippocampus, or prefrontal cortex, have been reported following depression $[7,4]$. The nucleotide-binding domain, leucine-rich repeat (NLR) pyrin domain protein 3 (NLRP3) inflammasomes, an intracellular multi-protein complex, is activated in various neurological and psychological disorders [8]. Full assembly and activation of the NLRP3 complex depend on two important factors of nuclear factor kappa B (NF-kB) signaling pathways and interaction of NLRP3 protein with the rest of the inflammasome machinery, including apoptosis-associated speck-like protein containing a caspase recruitment domain (ASC) and procaspase 1 [9]. After activation of complex, 
procaspase 1 is cleaved into caspase 1, which activates IL-1 $\beta$ from the pro-IL-1 [10]. A growing body of evidence confirmed that NF-KB is an important mediator of stress-induced neurological impairments [11].

Up to now, several animal models have been utilized to investigate the pathological events of the depression progression or effects of antidepressants. The models with exposure of the animal to stressors with their responses, which mimic human depression, are the most popular models [12]. Chronic restraint stress is a low-cost stressor that induces a series of behavioral changes similar to those seen in humans [13].

Different classes of antidepressants have been developed based on the hypothesis that depression, as a neuroendocrine disorder, is resulted from an imbalance in the monoamine neurotransmitters [14]. However, several side effects, e.g., sexual dysfunction, cardiovascular disease, and osteoporosis, have been reported for these antidepressants $[15,16]$. Recently, the relationship between thyroid axis abnormalities and psychiatric disorders has been proven [17]. Triiodothyronine (T3) and thyroxine (T4), two major thyroid hormones (THs), are secreted from the thyroid gland [18]. Both hyper and hypothyroidism patients experience numerous neuropsychiatric manifestations, such as anxiety and $\mathrm{e}$ depressive disorders [19]. Additionally, a "low T3 syndrome", decreased peripheral conversion of T4 to T3 in the normal production of $\mathrm{TH}$, has been described in patients with psychiatric depression [20]. THs treatment of patients with mood disorders, particularly depressive disorders, was reported in numerous studies [21]. It was also demonstrated that T3 could augment and accelerate treatment response with antidepressants [22]. T3 and T4 can cross the blood-brain barrier (BBB) via monocarboxylate 8 (MCT8), a specific transporter of THs [23]. The effects of T3 are mediated by its binding to TH receptor (TR) isoforms, predominantly TRa1 and TRß1. Notably, TRa1 is located on nearly all types of neurons [24]. Thus, the adequate local concentration of T3 is important for TRs activation [25]. T3 (the active and less $\mathrm{TH}$ ) contributes to numerous biologic functions in normal and pathologic conditions via different mechanisms. THs regulate the expression of oxidative stress products, glutamate, and neurotrophic factors in neurological disorders [26]. Furthermore, the anti-inflammatory effects of THs were reported in several studies $[27,28]$.

Due to the beneficial effects of T3, we used a single dose of intraventricular (IVC) T3 to evaluate its antidepressive effects in a chronic restraint stress model. To understand the mechanisms of effects of T3 as a therapeutic approach, we designed an experiment to examine: (1) depressive-like behaviors of animals, (2) activation of the NLRP3 inflammasome, and (3) pathological alterations via Nissl staining.

\section{Method And Material}

\section{Animals}

This research was approved by the Ethics Committee of Animal Experiments at Sadat City University, Sadat City, Egypt, funded by Taif University (Supporting Project number: TURSP-2020/99), and performed based on the Guide for the Care and Use of Laboratory Animals (8th edition, National Academies Press). 
In the present study, 36 male Wistar rats (weighing 150-220 g) were kept in the standard temperature $\left(23+1^{\circ} \mathrm{C}\right), 12 / 12 \mathrm{~h}$ light/ dark cycle with ad libitum access to food and water.

\section{Experimental design}

The study protocol was designed as demonstrated in Figure 1. Rats were randomly allocated to three groups ( $n=12$ in each group): Control (healthy animals), Model: with rats subjected to restraint stress and IVC injected with phosphate-buffered saline (PBS) [29], Model+T3: rats subjected to restraint stress and injected IP with T3. The rats in the Model and Model+T3 groups were exposed to stress. For this purpose, each rat was placed in a plastic cylinder ( $3 \mathrm{~h} /$ day from 8:00 am to 11:00 am) for 14 consecutive days [29]. Twenty-four hours after model induction, the animals in the Model+T3 group were injected with IVC T3 (a single dose of $25 \mathrm{ug} / \mathrm{kg}$ body). T3 was dissolved in dimethyl sulfoxideand diluted with PBS (a total volume of $5 \mathrm{ul}$ ) to be injected into the right ventricle via a Hamilton syringe (bregma: $\mathrm{ML}=-1.8 \mathrm{~mm}$, $\mathrm{AP}=-0.9 \mathrm{~mm}$, and $\mathrm{DV}=3.5 \mathrm{~mm}$ deep from the dura) [26]. The equal volume of PBS was injected into the right ventricle of rats in the Model group. The rats were examined via forced swimming (FST), tail suspension (TST), and open field (OFT) tests. In the end, the brains of rats were used for various molecular ( $n=8$ in each group) and histological ( $n=4$ in each group) studies. The fresh samples of the hippocampus were extracted immediately after sacrificing and put in a freezing tube and kept at $-80^{\circ}$. Also, for histological studies, the total brain was isolated after prefixation. The prefixation procedure was done via the transcranial perfusion of normal saline to remove the blood from the brain, followed by $4 \%$ paraformaldehyde perfusion (PFA, Sigma) via the same route. The post-fixed was done by using a $10 \%$ formalin at $4^{\circ} \mathrm{C}$ for $72 \mathrm{~h}$.

\section{Behavioral study}

\section{Forced swimming test (FST)}

A glass cylinder with a height of $80 \mathrm{~cm}$ and a diameter of $30 \mathrm{~cm}$ filled with $40 \mathrm{~cm}$ of water $\left(25^{\circ} \mathrm{C}\right)$ was used to evaluate the rats' behavior for two consecutive swimming sessions of training and test. The cylinder was filled with tap water $\left(23 \pm 1{ }^{\circ} \mathrm{C}\right)$, and water depth was adjusted according to the animal size. Therefore, rats could not touch the bottom of the cylinder. At first, for training, each rat was placed in the cylinder for 10 minutes and forced to swim. After $24 \mathrm{~h}$, the procedure was repeated for 5 min period as a test session. In this session, the animal behavior was video-recorded by a blind observer, and the immobility, latency, swimming, and climbing times were recorded [30]. An enhanced time of immobility time was considered as depressive-like behavior.

\section{Tail Suspension Test (TST)}

One day after FST, TST was performed. Briefly, each rat was suspended via its tails using adhesive tape to a horizontal bar for 6 min ( 2 min for adaptation and 4 min for test). The test was performed by a blind observer, and the immobility time was recorded. 


\section{Open Field Test (OFT)}

The OFT was performed to evaluate the depressive-like behavior, as reported previously [31]. Briefly, the open-field apparatus ( $80 \mathrm{~cm} \times 80 \mathrm{~cm} \times 50 \mathrm{~cm}^{2}$ ) is consists of floor divided into 25 equal squares. Each rat was placed in the center of the open field individually and allowed to freely explore for $5 \mathrm{~min}$, and the behavior was recorded. The time of immobility was evaluated for a 5 min period. After finishing the test, the rat was placed in the home cage by the experimenter. After each test, the apparatus was cleaned with $90 \%$ ethanol to remove olfactory cues.

\section{RNA Extraction and Quantitative Real-Time PCR (qRT-PCR)}

The fresh samples were used for quantitative real-time PCR (qRT-PCR) assay ( $n=4$ in each group) of NFKB, NLRP3, Caspase-1, and ASC. In summary, total RNA was isolated from samples by using the Tripure Isolation Reagent (Roche Applied Science, Peuzberg, Germany) according to the instruction. NanoDrop ${ }^{\mathrm{TM}}$ 2000/2000c spectrophotometer (Thermo Fisher Scientific) was used for evaluation of its purity. For complementary DNA synthesis, PrimeScript RT Reagent Kit (Takara Bio Inc., Otsu, Shiga, Japan) was used for reverse transcription. The PCR primers used are shown in Table 1. The StepOnePlus Real-Time PCR machine was carried out to run the reactions in triplicates. beta-actin ( $\beta$-actin) was used to normalize the gene expression, and the relative fold change expression of genes was calculated via the 2- $\Delta \Delta \mathrm{Ct}$ method [32].

\section{Western Blot}

The isolated hippocampal samples were used for western blot ( $n=4$ in each group) to determine the synthesis of inflammasome proteins. The samples were lysed via a lysis buffer (RIPA) and centrifuged. Total Protein Kit, Micro (Sigma, USA) was used to detect the total protein concentration. After protein denaturation, $5 \mu \mathrm{g}$ of protein was loaded on 10\% SDS-PAGE, and separated proteins were put on polyvinylidene difluoride transfer membranes (Sigma, USA) and then incubated for one hour with specific primary antibodies (Novus Biologicals, USA). Then, the procedure was followed by incubation of Membranes for one hour with anti-rabbit horseradish peroxidase (HRP)-conjugated secondary antibodies (Abcam, Germany). Protein bands were detected by the luminescent substrate solution (Sigma, USA). Finally, to quantify the specific bands, Image J software (NIH, USA) was used. GAPDH (Thermoscientific, USA) was used for normalization [33].

\section{Nissl Staining (Cresyl Violet Staining)}

After fixation and tissue processing ( $\mathrm{n}=4$ samples in each group), 5-um-thick coronal sections cut by microtome (Leica Biosystems, Milan, Italy) and mounted on the slides. After subjecting to Nissl staining, four random sections were picked for light microscopy observation. The photographs were prepared under an optical microscope (Labomed, USA). The photomicrographs were prepared from the CA1 region, and the number of dark neurons (intensely stained neurons) were calculated in each photomicrograph. 


\section{Statistical analysis}

Data were analyzed via SPSS software (V22.0) and expressed as mean \pm SD. The analyses were carried out using the one-way analysis of variance (ANOVA) and followed by post hoc Tukey's comparison. $P$ value less than 0.05 was considered as significant.

\section{Results}

\section{Behavioral tests}

To determine the effects of T3 on depressive-like behaviors, we performed behavioral investigations, including FST, TST, and OFT. Our results showed that exposure of animals to restraint stress (Model and Model+T3 groups) enhanced the immobility and latency time ( $P<0.05$, fig. 2 , a-b) and reduced the swimming and climbing time ( $P<0.05$, fig. $2, \mathrm{c}-\mathrm{d})$ in FST evaluations and treatment with T3 could improve the FST behaviors compared to the Model group $(P<0.05$, fig. 2 , a-d). Increased immobility times in TST and OFT were also seen in the Model and Model+T3 groups compared to the Control group $(P<0.05$, fig. 2 , e-f). Treatment of rats with T3 decreased the mean time of immobility in both tests compared to the Model group $(P<0.05$, fig. 2 , e-f).

\section{qRT-PCR analysis}

The mean gene expression of hippocampal NF-KB, NLRP3, ASC, and Caspase-1, was evaluated in each group. A significant increase in the gene expression of hippocampal NF-KB, NLRP3, ASC, and Caspase-1 was observed in the Model and Model+T3 groups compared to the Control group $(P<0.05$, fig. 3, a-d). The gene expression of hippocampal NF-KB, NLRP3, Caspase-1, and ASC was significantly reduced in the Model+T3 group compared to the Model group $(P<0.05$, fig. 3 , a-d $)$.

\section{Western Blot Analysis}

The mean protein levels of NF-KB, NLRP3, Caspase-1, and ASC were investigated in the hippocampal region. Fig. 4 shows that there was a significant increase in the protein levels of hippocampal NF-KB, NLRP3, ASC, and Caspase- 1 in the Model and Model+T3 groups compared to the Control group $(P<0.05$, fig. 4, a-e). The protein levels of hippocampal NLRP3, Caspase-1, ASC, and significantly reduced in the Model+T3 group compared with the Model group $(P<0.05$, fig. 4 , a-e).

\section{Histopathological Examination}

For the evaluation of pathological changes, Nissl staining was performed. The pathological alterations of CA1 were investigated in terms of the number of dark neurons and the pyramidal cell layer thickness. A significantly increased number of dark neurons in the CA1 region was reported in the Model and Model+T3 groups compared to the Control group $(P<0.05$, fig. 5, a-d). Also, the number of dark neurons reduced in the Model+T3 group compared to the Model group ( $P<0.05$, fig. 5, a, b, c, \& d). The pyramidal cell layer thickness significantly decreased in the CA1 region in the Model and Model+T3 groups 
compared to the Control group ( $P<0.05$, fig. 5, a, b , c, \& e). In addition, there was a significant increase in the pyramidal cell layer thickness in the Model+T3 group compared to the Model group $(P<0.05$, fig. 5 , a, b, c, \& e).

\section{Discussion}

The results of the current study revealed that treatment with a single dose of T3 ameliorated the depression-like behaviors induced by restraint stress. The IVC microinjection of T3 could alleviate the activity of inflammasome via reducing the gene expression and protein concentrations of NF-KB, NLRP3, ASC, and Caspase-1 in the hippocampus. Additionally, T3 improved the histopathological changes of the CA1 hippocampal region.

In the present study, we exposed the animals to chronic immobility stress to induce depressive-like behavior. According to the findings, chronic stress increased the immobility behavior of animals. Also, the enhanced levels of NF-KB, NLRP3, ACs, and Caspase-1 were observed in the hippocampus. Pathologically, the number of dark neurons and the pyramidal layer thickness decreased in the Model group. These findings indicated that neuroinflammation after animals to the stressor induced the histopathological and behavioral alterations in animals. Rodent models of chronic restraint stress with more than 35 years of use for modeling psychological disease have been employed in the investigation of biological and behavioral manifestations of clinical depressive disorders in humans [34,35]. Depression is a psychological disorder associated with several pathological events, such as neural death in the hippocampus [36]. The neural loss in the hippocampus is an important factor in decreasing the hippocampal volume in depressive disorders [37]. According to the literature, psychological or physical stressors may be followed by the activation of inflammation and subsequent production of inflammatory cytokines, resulting in functional and structural changes in neurons [38]. Several studies have demonstrated that the interaction between the CNS and the immune system plays a critical role in stressinduced neuroinflammation and depression [39]. Therefore, peripheral administration of immunostimulants in animal models is often used for the induction of inflammation-related depression [40]. The anxious and depressive behaviors have been shown to be associated with the enhanced levels of TNF-a in the hippocampus and striatum [41].

Among a wide range of cytokines, IL-1 $\beta$ seems to play an important role in the pathological features of depressive-like behavior caused by stress [42]. It has been demonstrated that IL-1 $\beta$ reduced the neurogenesis in human hippocampal progenitor cells through stimulation of the kynurenine signaling pathway, a common finding in depression. Both inhibitors of this pathway and traditional antidepressants can modulate this effect [43]. The NLRP3 inflammasome is the major intercellular mediator of IL-1 $\beta$ maturation and secretion [44]. The activation the NF- $K B$ pathway and NLRP3 inflammasome has been proven in animal models of depression and patients with major depressive disorders $[45,46]$. Furthermore, NF- $k$ B signaling and NLRP3 inflammasome have previously been suggested as a link between the immune system and stressors, the potential pathologic features in the development of depressive disorder $[14,46]$. Activation of NF-KB induces the transcription of pro-IL-18, 
pro-IL-1 $\beta$, and NLRP3 [47]. Besides, some factors, e.g., overload calcium, reactive oxygen species, ATP can directly stimulate NLRP3 inflammasome [48, 49]. On the other hand, inflammation is commonly related to oxidative stress. It seems that oxidative damage, such as DNA oxidation and lipid peroxidation, contribute to the pathological features of neuropsychiatric diseases [50]. Additionally, the association between glutamate and inflammation has been well demonstrated [35], as a large number of clinical studies suggest that pathophysiology of depression is associated with dysfunction of the predominant glutamatergic system, known as the glutamate hypothesis [51]. The NLRP3 inflammasome also is responsible for pyroptosis and apoptosis in hippocampus neurons mediates depressive-like behavior in diabetic mice [52]. Therefore, the increased number of dark neurons are associated with enhanced activation of NF-KB/ NLRP3 inflammasome pathway.

In the current study, we used a single dose of IVC T3 following the induction of depressive-like behaviors. The administration of T3 could inhibit the activation of the inflammasome complex in the hippocampal region via reducing the NF-KB, NLRP3, ASC, and Caspase- 1 concentration and prevent the pathological features seen in the hippocampal region. These alterations were correlated with improved depressive-like behavior according to FST, TST, and OFT findings, suggesting that T3 could survive the neurons of the CA1 region via anti-inflammatory properties. According to the evidence from preclinical and clinical THs, abnormalities are seen in depressive disorders $[53,54]$. Therefore, the regulation of the thyroid axis may help to improve the symptoms of depression. T3 alone or in combination with antidepressants has been used for the treatment of patients with depressive disorders [54]. THs are essential for brain development in both fetal and post-natal periods [24]. As previously recorded in mammalian brain researches, the levels of T3 and T3/T4 ratio are higher in the brain compared to the systemic circulation [55]. T3 has been shown to augment or accelerate the treatment of depressive disorders [56]. This hormone acts as a stimulator of gene expression and regulates the cellular energy for metabolism and neurogenesis in the CNS [57]. Recently, IVC injection of T3 was revealed to survive neurons of the CA1 hippocampal region in the rat model of brain stroke via the upregulation of neurotrophic factors [58]. In another study, exogenous T3 exerted neuroprotective features via modulation of the NF-KB pathway (anti-inflammatory effects) and enhanced levels of neurotrophic factors in a traumatic brain injury [59]. In addition, the administration of T3 could diminish alcoholic liver disease and ischemia-reperfusion-induced liver via modulation of the NLRP3 signaling pathway $[60,61]$. In accordance with our results, these findings confirm that T3 of may suppress the NF-KB/ NLRP3 inflammasome pathway via anti-inflammatory features, contribute to reverses the neural death in hippocampus, and improve the depressive like behavior in rats exposure to the chronic stress.

\section{Conclusion}

In summary, restraint stress induction activated the NLRP3 inflammasome in the hippocampus region, correlated with pathological and behavioral alterations. To attenuate these changes, we used a single dose of T3 via the IVC route. The findings of the current study indicated that T3 regulated the gene expression and protein levels of NF-KB, NLRP3, ASC, and Caspase-1 in the hippocampus and improved the pathological and behavioral alterations induced by restraint stress in the rat model. According to the 
results, T3 could survive the pyramidal neurons of the hippocampus via regulation of e NF-KB/ NLRP3 inflammasome pathway and confirm the beneficial effects of T3 in the treatment of depressive symptoms.

\section{Declarations}

\section{Acknowledgments:}

This study was funded by Taif University, Taif, Saudi Arabia (Researchers supporting project number: TURSP-2020/99).

\section{Ethics approval and consent to participate:}

This research was approved by the Ethics Committee of Animal Experiments at Sadat City University, Sadat City, Egypt and performed based on the Guide for the Care and Use of Laboratory Animals (8th edition, National Academies Press).

\section{Consent for publication:}

$\mathrm{N} / \mathrm{A}$

\section{Competing interests:}

The authors declare that they have no competing interests.

\section{Funding:}

This study was funded by Taif University, Taif, Saudi Arabia (Researchers supporting project number: TURSP-2020/99).

\section{Availability of data and material:}

Data are however available from the authors upon reasonable request

\section{Authors' contributions:}

LH and AME contributed substantially to the conception and design of the study. AME, and TM contributed to perform the experiment, LH contributed to analyze the data, TM and AME drafted or provided critical revision of the article. LH provided the final approval of the version to publish. All authors discussed the results and contributed to the final manuscript.

\section{References}

1. Joo Y, Choi KM, Lee YH, Kim G, Lee DH, Roh GS et al (2009) Chronic immobilization stress induces anxiety- and depression-like behaviors and decreases transthyretin in the mouse cortex. Neurosci Lett 
461(2):121-125. doi:https://doi.org/10.1016/j.neulet.2009.06.025

2. Kim YR, Park B-K, Kim YH, Shim I, Kang I-C, Lee MY. Antidepressant effect of fraxinus rhynchophylla hance extract in a mouse model of chronic stress-induced depression. BioMed Research International. 2018;2018

3. Ning J, Jing-Wei L, Hai-Xia W, Hong H, Qiong W, Shan-Guang C et al (2019) Antidepressant-like Effects of Ginsenoside Rg1 in the Chronic Restraint Stress-induced Rat Model. Digital Chinese Medicine 2(4):207-218. doi:https://doi.org/10.1016/j.dcmed.2020.01.002

4. Mokhtari T, Tu Y, Hu L (2019) Involvement of the hippocampus in chronic pain and depression. Brain Science Advances 5(4):288-298

5. Anisman H (2009) Cascading effects of stressors and inflammatory immune system activation: implications for major depressive disorder. J Psychiatry Neurosci 34(1):4-20

6. Johnson JD, Barnard DF, Kulp AC, Mehta DM (2019) Neuroendocrine Regulation of Brain Cytokines After Psychological Stress. Journal of the Endocrine Society 3(7):1302-1320. doi:10.1210/js.201900053

7. Johnson JD, Barnard DF, Kulp AC, Mehta DM (2019) Neuroendocrine Regulation of Brain Cytokines After Psychological Stress. Journal of the Endocrine Society 3(7):1302-1320. doi:10.1210/js.201900053

8. Bazrafkan M, Nikmehr B, Shahverdi A, Hosseini SR, Hassani F, Poorhassan M et al (2018) Lipid Peroxidation and Its Role in the Expression of NLRP1a and NLRP3 Genes in Testicular Tissue of Male Rats: a Model of Spinal Cord Injury. Iran Biomed J 22(3):151-159. doi:10.22034/ibj.22.3.151

9. Mohammed I, Ijaz S, Mokhtari T, Gholaminejhad M, Mahdavipour M, Jameie B et al. Subventricular zone-derived extracellular vesicles promote functional recovery in rat model of spinal cord injury by inhibition of NLRP3 inflammasome complex formation. Metabolic Brain Disease. 2020:1-10

10. Broz P, Dixit VM (2016) Inflammasomes: mechanism of assembly, regulation and signalling. Nature reviews Immunology 16(7):407-420. doi:10.1038/nri.2016.58

11. Koo JW, Russo SJ, Ferguson D, Nestler EJ, Duman RS (2010) Nuclear factor-kappaB is a critical mediator of stress-impaired neurogenesis and depressive behavior. Proc Natl Acad Sci U S A 107(6):2669-2674. doi:10.1073/pnas.0910658107

12. Ito $\mathrm{N}$, Hirose E, Ishida T, Hori A, Nagai T, Kobayashi $Y$ et al (2017) Kososan, a Kampo medicine, prevents a social avoidance behavior and attenuates neuroinflammation in socially defeated mice. $\mathrm{J}$ Neuroinflamm 14(1):1-15

13. Xu P, Wang K, Lu C, Dong L, Chen Y, Wang Q et al (2017) Effects of the chronic restraint stress induced depression on reward-related learning in rats. Behav Brain Res 321:185-192

14. Chávez-Castillo M, Núñez V, Nava M, Ortega Á, Rojas M, Bermúdez V et al (2019) Depression as a Neuroendocrine Disorder: Emerging Neuropsychopharmacological Approaches beyond Monoamines. Adv Pharmacol Sci 2019:7943481-. doi:10.1155/2019/7943481

15. Nahas ARF, Sulaiman SAS (2017) Increased Risk of Osteoporosis in Depressive Patients with Erectile Dysfunction: A Cross-sectional Study from Malaysia. J Pharm Bioallied Sci 9(3):178-184. 
doi:10.4103/jpbs.JPBS_64_17

16. Hosseinzadeh Zoroufchi B, Doustmohammadi H, Mokhtari T, Abdollahpour A (2020)

Benzodiazepines related sexual dysfunctions: A critical review on pharmacology and mechanism of action. Revista internacional de andrologia. doi:10.1016/j.androl.2019.08.003

17. Joffe RT (2011) Hormone treatment of depression. Dialogues Clin Neurosci 13(1):127-138

18. Hage MP, Azar ST. The link between thyroid function and depression. Journal of thyroid research. $2012 ; 2012$

19. Hage MP, Azar ST (2012) The Link between Thyroid Function and Depression. Journal of thyroid research 2012:590648-. doi:10.1155/2012/590648

20. Premachandra BN, Kabir MA, Williams IK (2006) Low T3 syndrome in psychiatric depression. J Endocrinol Investig 29(6):568-572. doi:10.1007/bf03344150

21. Duval F (2018) Thyroid hormone treatment of mood disorders. Current Treatment Options in Psychiatry 5(4):363-376

22. Parmentier T, Sienaert $P$ (2018) The use of triiodothyronine (T3) in the treatment of bipolar depression: A review of the literature. J Affect Disord 229:410-414. doi:https://doi.org/10.1016/j.jad.2017.12.071

23. Rodrigues TB, Ceballos A, Grijota-Martínez C, Nuñez B, Refetoff S, Cerdán S et al (2013) Increased oxidative metabolism and neurotransmitter cycling in the brain of mice lacking the thyroid hormone transporter SLC16A2 (MCT8). PLoS One 8(10):e74621

24. Schroeder AC, Privalsky ML (2014) Thyroid hormones, $t 3$ and t4, in the brain. Front Endocrinol (Lausanne) 5:40

25. Schroeder AC, Privalsky ML (2014) Thyroid hormones, t3 and t4, in the brain. Front Endocrinol (Lausanne) 5:40-. doi:10.3389/fendo.2014.00040

26. Mokhtari T, Akbari M, Malek F, Kashani IR, Rastegar T, Noorbakhsh F et al (2017) Improvement of memory and learning by intracerebroventricular microinjection of $\mathrm{T} 3$ in rat model of ischemic brain stroke mediated by upregulation of BDNF and GDNF in CA1 hippocampal region. Daru 25(1):4-. doi:10.1186/s40199-017-0169-x

27. Panveloski-Costa A, Silva Teixeira S, Ribeiro I, Serrano-Nascimento C, Das Neves R, Favaro R et al (2016) Thyroid hormone reduces inflammatory cytokines improving glycaemia control in alloxaninduced diabetic wistar rats. Acta Physiol 217(2):130-140

28. Panveloski-Costa AC, Serrano-Nascimento C, Bargi-Souza P, Poyares LL, Viana GdS, Nunes MT (2018) Beneficial effects of thyroid hormone on adipose inflammation and insulin sensitivity of obese Wistar rats. Physiol Rep 6(3):e13550. doi:10.14814/phy2.13550

29. Guedri K, Frih H, Chettoum A, Rouabhi R (2017) Chronic restraint stress induced neurobehavioral alterations and histological changes in rat. Toxicology Environmental Health Sciences 9(2):123-129. doi:10.1007/s13530-017-0312-6 
30. Porsolt RD (2000) Animal models of depression: utility for transgenic research. Rev Neurosci 11(1):53-58. doi:10.1515/revneuro.2000.11.1.53

31. Belviranlı M, Okudan N (2015) The effects of Ginkgo biloba extract on cognitive functions in aged female rats: the role of oxidative stress and brain-derived neurotrophic factor. Behav Brain Res 278:453-461

32. Livak KJ, Schmittgen TD (2001) Analysis of relative gene expression data using real-time quantitative PCR and the $2-\Delta \Delta C T$ method. methods 25(4):402-408

33. ljaz S, Mohammed I, Gholaminejhad M, Mokhtari T, Akbari M, Hassanzadeh G (2020) Modulating pro-inflammatory cytokines, tissue damage magnitude, and motor deficit in spinal cord injury with subventricular zone-derived extracellular vesicles. J Mol Neurosci 70(3):458-466

34. Keim KL, Sigg EB. Plasma corticosterone and brain catecholamines in stress: effect of psychotropic drugs. Pharmacology, biochemistry, and behavior. 1977;6(1):79-85. doi:10.1016/00913057(77)90162-9

35. McWhirt J, Sathyanesan M, Sampath D, Newton SS (2019) Effects of restraint stress on the regulation of hippocampal glutamate receptor and inflammation genes in female C57BL/ 6 and BALB/c mice. Neurobiology of Stress 10:100169. doi:https://doi.org/10.1016/j.ynstr.2019.100169

36. Lee AL, Ogle WO, Sapolsky RM (2002) Stress and depression: possible links to neuron death in the hippocampus. Bipolar disorders 4(2):117-128. doi:10.1034/j.1399-5618.2002.01144.x

37. Sapolsky RM (2000) The possibility of neurotoxicity in the hippocampus in major depression: a primer on neuron death. Biol Psychiatry 48(8):755-765. doi:10.1016/s0006-3223(00)00971-9

38. Voorhees JL, Tarr AJ, Wohleb ES, Godbout JP, Mo X, Sheridan JF et al (2013) Prolonged restraint stress increases IL-6, reduces IL-10, and causes persistent depressive-like behavior that is reversed by recombinant IL-10. PLoS One 8(3):e58488-e. doi:10.1371/journal.pone.0058488

39. Grippo AJ, Scotti M-AL. Stress and neuroinflammation. Inflammation in Psychiatry. Karger Publishers; 2013. p. 20-32

40. Dang R, Guo Y-y, Zhang K, Jiang P, Zhao M (2019) -g. Predictable chronic mild stress promotes recovery from LPS-induced depression. Mol Brain 12(1):1-12

41. Zhou R, Wang F, Zhao G, Xia W, Peng D, Mao R et al (2018) Effects of tumor necrosis factor-a polymorphism on the brain structural changes of the patients with major depressive disorder. Translational psychiatry 8(1):1-9

42. Goshen I, Kreisel T, Ben-Menachem-Zidon O, Licht T, Weidenfeld J, Ben-Hur T et al (2008) Brain interleukin-1 mediates chronic stress-induced depression in mice via adrenocortical activation and hippocampal neurogenesis suppression. Molecular psychiatry 13(7):717-728

43. Zunszain PA, Anacker C, Cattaneo A, Choudhury S, Musaelyan K, Myint AM et al (2012) Interleukin$1 \beta$ : a new regulator of the kynurenine pathway affecting human hippocampal neurogenesis. Neuropsychopharmacology: official publication of the American College of Neuropsychopharmacology 37(4):939-949. doi:10.1038/npp.2011.277 
44. Kelley N, Jeltema D, Duan Y, He Y (2019) The NLRP3 Inflammasome: An Overview of Mechanisms of Activation and Regulation. Int J Mol Sci 20(13):3328. doi:10.3390/ijms20133328

45. Stepanichev M, Dygalo NN, Grigoryan G, Shishkina GT, Gulyaeva N. Rodent models of depression: neurotrophic and neuroinflammatory biomarkers. BioMed research international. 2014;2014

46. Wang M, Yan S, Zhou Y, Xie P. <i > trans-Cinnamaldehyde Reverses Depressive-Like Behaviors in Chronic Unpredictable Mild Stress Rats by Inhibiting NF- K B/NLRP3 Inflammasome Pathway. Evidence-Based Complementary and Alternative Medicine. 2020;2020:4572185. doi:10.1155/2020/4572185.

47. Liu T, Zhang L, Joo D, Sun S-C (2017) NF-kB signaling in inflammation. Signal Transduct Target Ther 2:17023. doi:10.1038/sigtrans.2017.23

48. Lee G-S, Subramanian N, Kim Al, Aksentijevich I, Goldbach-Mansky R, Sacks DB et al (2012) The calcium-sensing receptor regulates the NLRP3 inflammasome through Ca $2+$ and cAMP. Nature 492(7427):123-127

49. Heid ME, Keyel PA, Kamga C, Shiva S, Watkins SC, Salter RD (2013) Mitochondrial reactive oxygen species induces NLRP3-dependent lysosomal damage and inflammasome activation. J Immunol 191(10):5230-5238

50. Cheng J, Dong S, Yi L, Geng D, Liu Q (2018) Magnolol abrogates chronic mild stress-induced depressive-like behaviors by inhibiting neuroinflammation and oxidative stress in the prefrontal cortex of mice. Int Immunopharmacol 59:61-67

51. Sanacora G, Treccani G, Popoli M (2012) Towards a glutamate hypothesis of depression: an emerging frontier of neuropsychopharmacology for mood disorders. Neuropharmacology 62(1):6377. doi:10.1016/j.neuropharm.2011.07.036

52. Li DX, Wang CN, Wang Y, Ye CL, Jiang L, Zhu XY et al (2020) NLRP3 inflammasome-dependent pyroptosis and apoptosis in hippocampus neurons mediates depressive-like behavior in diabetic mice. Behav Brain Res 391:112684. doi:10.1016/j.bbr.2020.112684

53. Lang UE, Borgwardt S (2013) Molecular mechanisms of depression: perspectives on new treatment strategies. Cell Physiol Biochem 31(6):761-777

54. Touma KTB, Zoucha AM, Scarff JR (2017) Liothyronine for Depression: A Review and Guidance for Safety Monitoring. Innov Clin Neurosci 14(3-4):24-29

55. Guadaño-Ferraz A, Escámez MJ, Rausell E, Bernal J (1999) Expression of type 2 iodothyronine deiodinase in hypothyroid rat brain indicates an important role of thyroid hormone in the development of specific primary sensory systems. J Neurosci 19(9):3430-3439

56. Rosenthal LJ, Goldner WS, O'Reardon JP (2011) T3 augmentation in major depressive disorder: safety considerations. Am J Psychiatry 168(10):1035-1040

57. Gothié JD, Sébillot A, Luongo C, Legendre M, Nguyen Van C, Le Blay K et al (2017) Adult neural stem cell fate is determined by thyroid hormone activation of mitochondrial metabolism. Mol Metab 6(11):1551-1561. doi:10.1016/j.molmet.2017.08.003 
58. Mokhtari T, Akbari M, Malek F, Kashani IR, Rastegar T, Noorbakhsh F et al (2017) Improvement of memory and learning by intracerebroventricular microinjection of T3 in rat model of ischemic brain stroke mediated by upregulation of BDNF and GDNF in CA1 hippocampal region. DARU Journal of Pharmaceutical Sciences 25(1):4

59. Crupi R, Paterniti I, Campolo M, Di Paola R, Cuzzocrea S, Esposito E (2013) Exogenous T3 administration provides neuroprotection in a murine model of traumatic brain injury. Pharmacological research 70(1):80-89. doi:10.1016/j.phrs.2012.12.009

60. Vargas R, Videla LA (2017) Thyroid hormone suppresses ischemia-reperfusion-induced liver NLRP3 inflammasome activation: Role of AMP-activated protein kinase. Immunology letters 184:92-97

61. Dong X, Yang H, Li C, Liu Q, Bai Q, Zhang Z (2018) Triiodothyronine alleviates alcoholic liver disease injury through the negative regulation of the NLRP3 signaling pathway. Experimental therapeutic medicine 16(3):1866-1872

\section{Tables}

Table 1. List of primers

\begin{tabular}{|lll|}
\hline Gene & Forward primer & Reverse Primer \\
NF-kB & AATTGCCCCCGGGCAAT & TCCCGTAAACCGGCGTAA \\
NLRP3 & GGAGTGGATAGGTTTGCTGG & GGTGTAGGGTCTGTTGAGGT \\
Caspase-1 & GTGGAGAGAAAGAAGGAGTGGT & GATGAGTGACTGAATGAAGAGG \\
ASC & TCTGGAGGGGTATGGCTTGG & GAGTGCTTGCCTGTGTTGGT \\
b-actin & ACAACCTTCTTGCAGCTCCTC & CTGACCCATACCCACCATCAC \\
\hline
\end{tabular}

\section{Figures}


T3 Injection

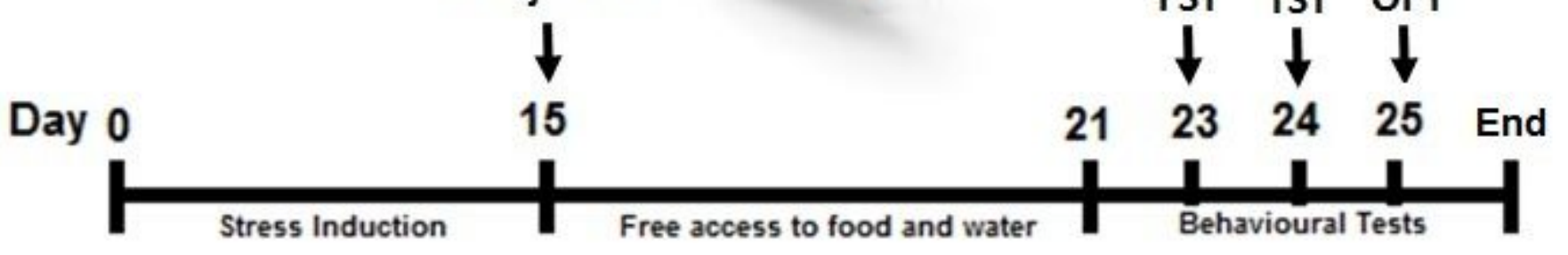

Figure 1

Study protocol. 

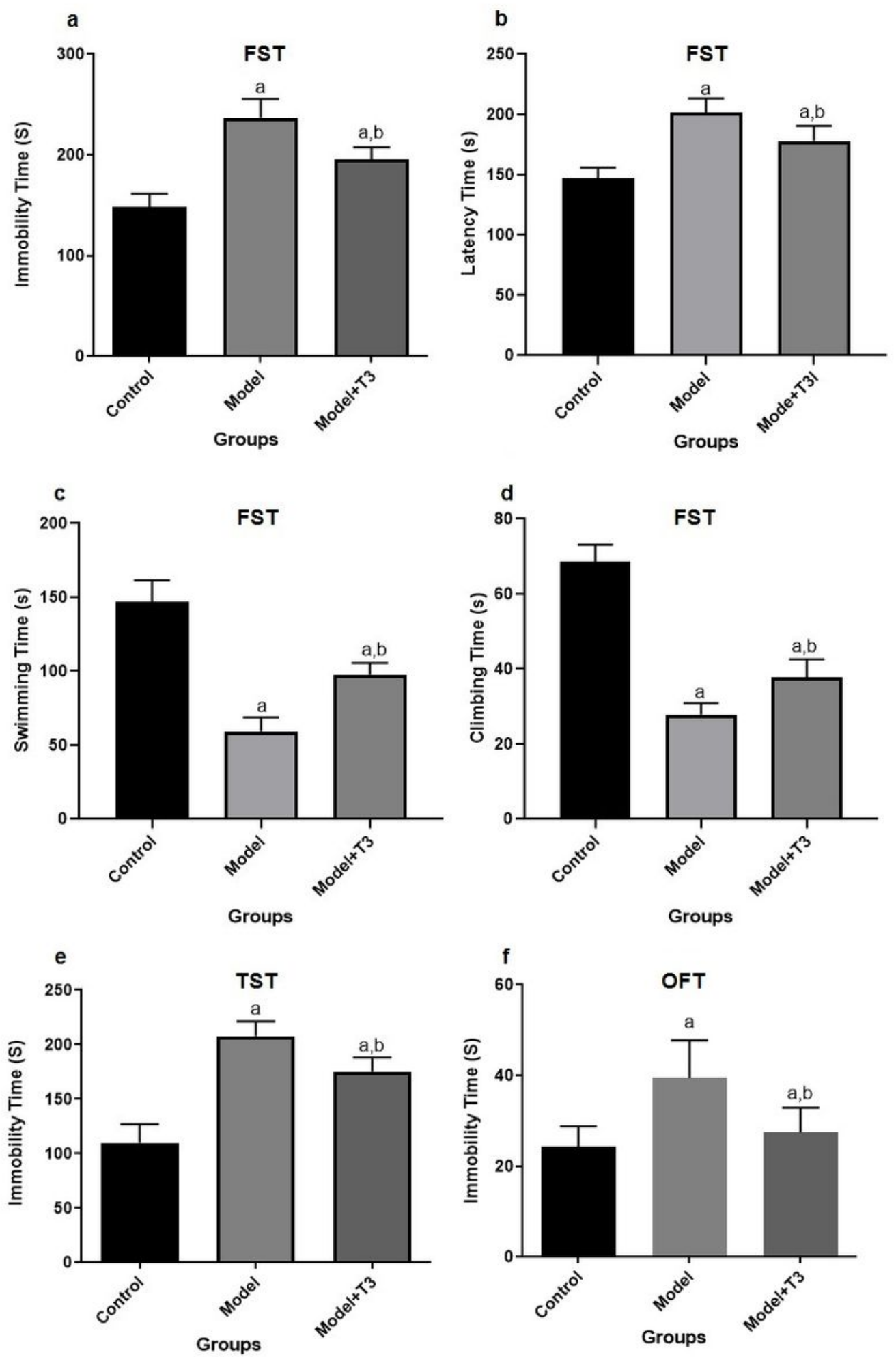

Figure 2

Effects of T3 on depressive-like behavior following restraint stress in rats. a. Immobility time (s), b. Latency time (s), c. Swimming time (s), and d. Climbing time (s) in FST, e. Immobility time (s) in TST, and f. Immobility time (s) in OFT in. a P< 0.05 compared to the Control group, b $\mathrm{P}<0.05$ compared to the Model group. Control: Normal and healthy rats, Model: Restraint stress group received PBS, Model+T3: Restraint stress group received T3. 

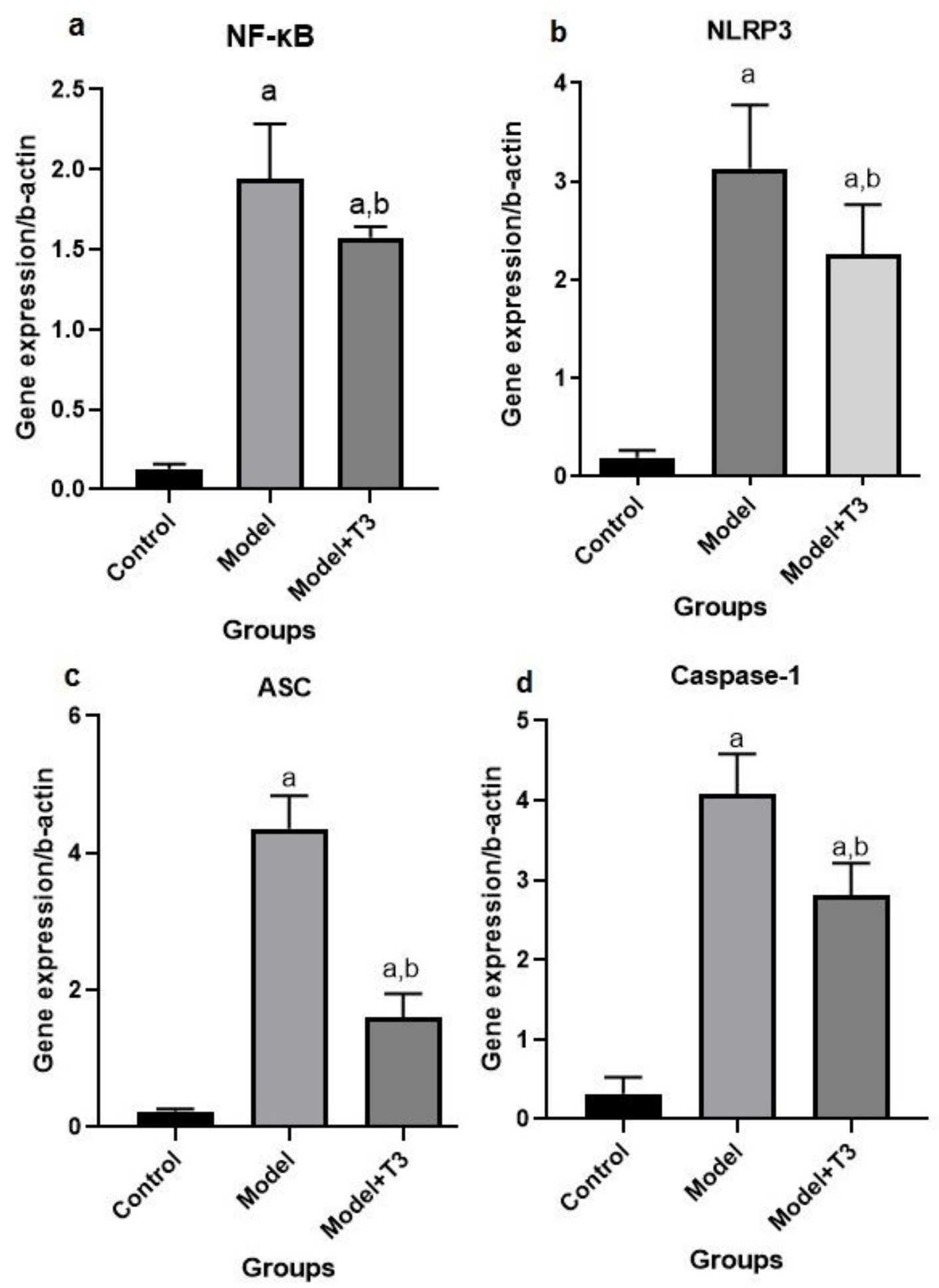

Figure 3

Effects of T3 on gene expression of a. NF-KB, b. NLRP3, c. Caspase-1, and d. ASC in the hippocampus of rats with depressive-like behavior. a $\mathrm{P}<0.05$ compared to the Control group, $\mathrm{b} \mathrm{P}<0.05$ compared to the Model group. Control: Normal and healthy rats, Model: Restraint stress group received PBS, Model+T3: Restraint stress group received T3. 


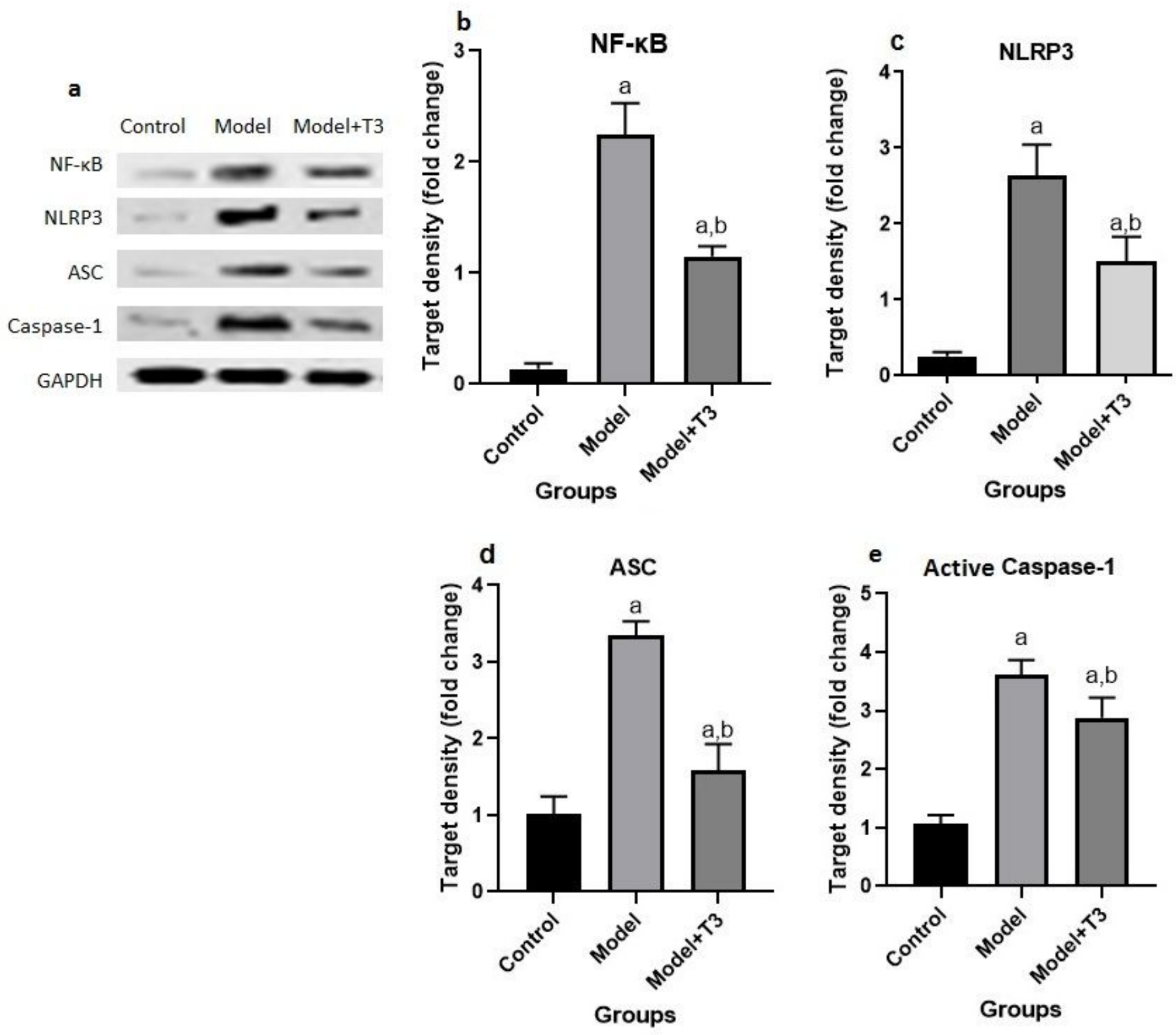

Figure 4

Effects of T3 on protein levels of a, b. NF-KB, a, c. NLRP3, a, d. Caspase-1, and a, e. ASC in the hippocampus of rats with depressive-like behavior. a $\mathrm{P}<0.05$ compared to the Control group, b $\mathrm{P}<0.05$ compared to the Model group. Control: Normal and healthy rats, Model: Restraint stress group received PBS, Model+T3: Restraint stress group received T3. 


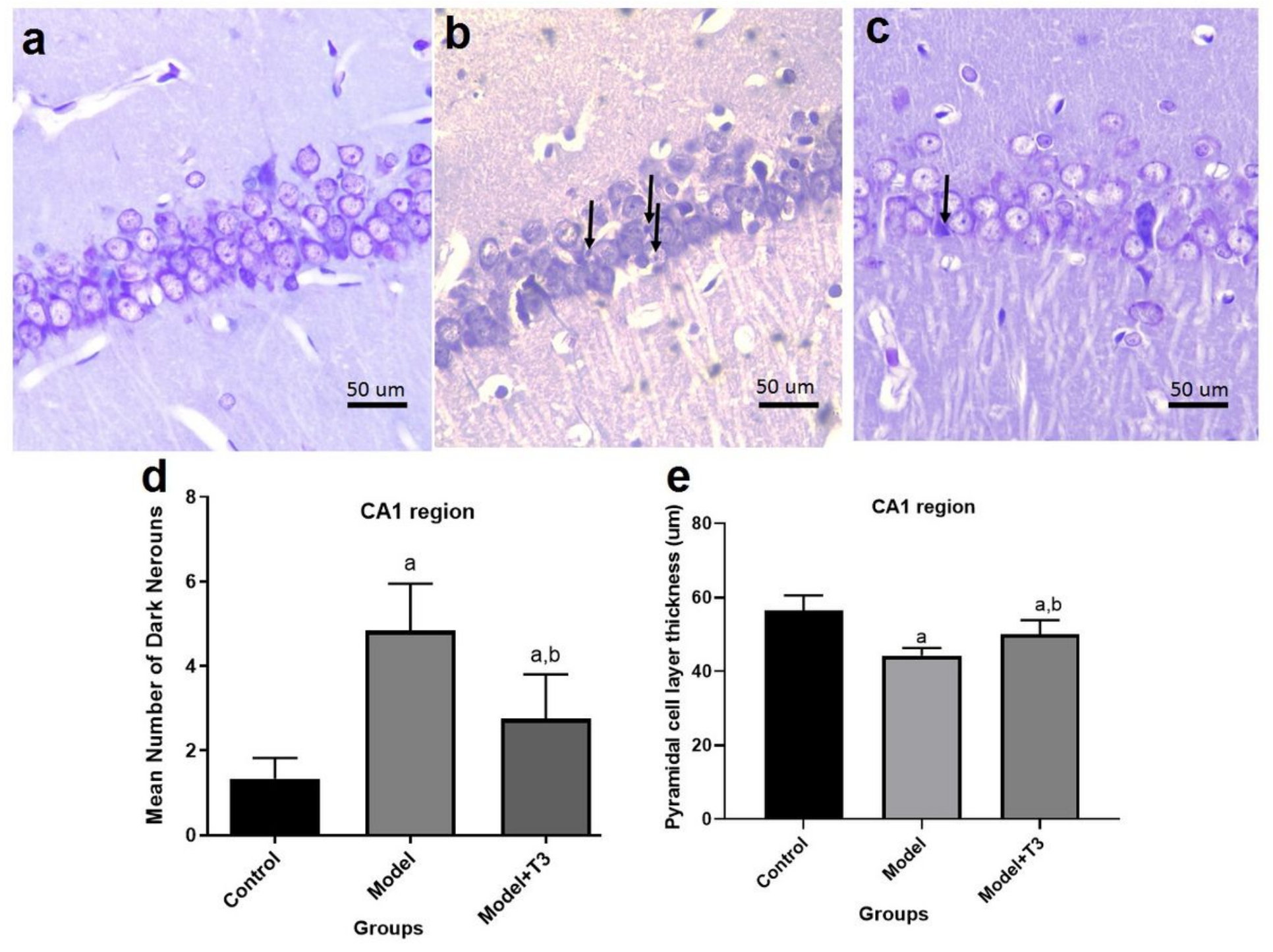

Figure 5

Effects of T3 on histopathological changes of the CA1 region of the hippocampus of mice with depressive-like behavior. a, b, c. Nissl staining (scale bar: $50 \mu \mathrm{m}$ ), d. Comparing the mean number of dark neurons (arrows) of the CA1 region in different groups. a $\mathrm{P}<0.05$ compared to the Control group, b $\mathrm{P}<$ 0.05 compared to the Model group. Control: Normal and healthy rats, Model: Restraint stress group received PBS, Model+T3: Restraint stress group received T3. 